\title{
hnRNP U interacts with the c-Myc-Max complex on the E-box promoter region inducing the ornithine decarboxylase gene
}

\author{
YOICHIRO MATSUOKA, NORIHISA UEHARA and AIRO TSUBURA
}

\begin{abstract}
Second Department of Pathology, Kansai Medical University, 10-15 Fumizono-cho, Moriguchi, Osaka 570-8506, Japan
\end{abstract}
Received January 28, 2009; Accepted March 13, 2009

DOI: $10.3892 /$ or_00000431

\begin{abstract}
The promoter of the ornithine decarboxylase $(O D C)$ gene contains two E-boxes, which are critical sites for transcriptional activation by the binding of c-Myc-Max heterodimers. We have identified heterogeneous nuclear ribonuclear protein $\mathrm{U}$ (hnRNP $\mathrm{U}$ ) as a component of the complex formed on the E-box-containing promoter region of the $O D C$ gene by using DNA-affinity chromatography, immunoprecipitation and chromatin immunoprecipitation assays. The N-terminal domain of hnRNP U was responsible for the association with c-Myc-Max complex. Down-regulation of hnRNP U with RNA interference blocked the induction of the $O D C$ gene and cell growth by serum stimulation, suggesting that hnRNP $U$ is a coactivator of the c-Myc-Max complex and essential for cell proliferation. Electrophoretic mobility-shift assays revealed that the segment between the two E-boxes in the promoter is the primary binding site of hnRNP U. The putative binding sequence was narroweddown to a 13-nucleotide segment by comparing the sequence between the E-boxes with the binding sites of hnRNP U, which were recently identified in the promoter of Bmall, a core component of the circadian molecular oscillator. These findings increase our knowledge of how the c-Myc-Max complex exerts its transcriptional regulatory role and suggest that hnRNP U may be a coactivator of this transcriptional activator complex.
\end{abstract}

\section{Introduction}

Heterogeneous nuclear ribonucleoprotein U (hnRNP U) is enriched in the nuclear matrix and regulates gene transcription (1-3). hnRNP U binds conserved AT-rich regions within the genome called scaffold attachment regions, which allow chromatin to attach to the nuclear matrix and form $\sim 5-200-\mathrm{kb}$ loops (4). hnRNP U was independently identified

Correspondence to: Dr Yoichiro Matsuoka, Second Department of Pathology, Kansai Medical University, 10-15 Fumizono-cho, Moriguchi, Osaka 570-8506, Japan

E-mail: matsuoyo@takii.kmu.ac.jp

Key words: c-Myc, E-box, heterogeneous nuclear ribonucleoprotein $\mathrm{U}$ as a protein that binds to these scaffold attachment region elements and was termed scaffold attachment factor A (5).

The multifunctional properties of hnRNP $U$ have been mapped to specific domains within the protein. The N-terminal domain (acidic and glutamine rich) is important for interactions with nuclear matrix and chromatin, and the $\mathrm{C}$-terminal domain, which contains an RGG box, is important for interactions with other hnRNP proteins to form hnRNP particles $(6,7)$. The middle domain mediates the interaction of hnRNP U with RNA polymerase II, and its association inhibits TFIIHmediated phosphorylation and RNA polymerase II elongation (8).

Myc proteins are basic helix-loop-helix leucine-zipper (bHLH-Zip) transcription factors whose function relies on heterodimerization with Max through their related bHLH-Zip domain (9). The Myc-Max heterodimer binds to E-box DNA sequences (CAYGTG) and can activate transcription through a tethered complex of proteins that contains histone acetyltransferase and other activities that remodel chromatin (10). Max also heterodimerizes with other proteins that contain Myc-like bHLH-Zip domains, including the Mad family proteins Mxd 1-4, the Mad-related protein Mnt, and Mga $(11,12)$. A current model proposes that E-boxes are occupied by Mnt-Max in quiescent cells, whereas Mnt-Max complexes are displaced by Myc-Max complexes in growth-stimulated cells $(13,14)$.

Since we previously proposed possible higher-order interactions among c-Myc, Max, HDAC1 and Mnt that regulate the expression of Myc-target genes such as the ornithine decarboxylase $(O D C)$ gene (15), we attempted to isolate other candidate molecules that might be involved in the regulation of gene expression by binding to the E-boxcontaining promoter region. During the study, we found interaction between hnRNP $\mathrm{U}$ and c-Myc-Max complexes, and a coactivator function of hnRNP $U$ for c-Myc-Max transcriptional activator.

\section{Materials and methods}

DNA-affinity chromatography and mass spectrometry. Nuclear extracts of rat $3 \mathrm{Y} 1$ fibroblasts were prepared by using NEPER nuclear and cytoplasmic extraction reagents (Pierce Biotechnology), according to the manufacturer's instructions. Extracts were pre-cleared by incubation with streptavidin magnetic beads (Takara Biotechnology, Ohtsu, Japan) in the presence of $50 \mathrm{ng} / \mathrm{ml}$ of salmon sperm DNA. The extracts $(100 \mu \mathrm{g})$ were incubated with a biotin-labeled oligonucleotide 
consisting of 62 bps of the E-box-containing region of the $O D C$ promoter (800 pmole) in $100 \mu 1$ of binding solution (20 mM Tris-HCl pH 7.9, $60 \mathrm{mM} \mathrm{KCl,} 1 \mathrm{mM}$ EDTA, $0.1 \%$ Triton X-100, $1 \mathrm{mM}$ DTT, $10 \mu \mathrm{g} / \mathrm{ml}$ leupeptin) for $20 \mathrm{~min}$ at room temperature, and the protein-DNA complexes were collected by incubation with $100 \mu 1$ of streptavidin magnetic beads for $1 \mathrm{~h}$ at $4^{\circ} \mathrm{C}$. After washing the beads 5 times with $1 \mathrm{ml}$ of the binding solution, the bound proteins were eluted with $50 \mu \mathrm{l}$ of elution buffer $(20 \mathrm{mM}$ Tris- $\mathrm{HCl} \mathrm{pH} 6.8,1 \mathrm{M}$ $\mathrm{NaCl}, 1$ mM EDTA, 1 mM DTT).

A band was excised from the silver stained gel, cut into pieces, washed twice with $200 \mu \mathrm{l}$ of $50 \mathrm{mM} \mathrm{NH} \mathrm{NCO}_{3}$ in $50 \%$ acetonitrile (v/v), and dehydrated with $100 \mu 1$ of $100 \%$ acetonitrile. Reduction of the protein sample was achieved with $10 \mathrm{mM} \mathrm{DTT} / 100 \mathrm{mM} \mathrm{NH} \mathrm{HCO}_{3}$ at $56^{\circ} \mathrm{C}$ for $1 \mathrm{~h}$, and alkylation was performed with $55 \mathrm{mM}$ iodoacetamide/100 mM $\mathrm{NH}_{4} \mathrm{HCO}_{3}$ for $45 \mathrm{~min}$ in the dark at room temperature. Subsequently, the gel pieces were washed twice for $5 \mathrm{~min}$ alternatively with $100 \mathrm{mM}$ ammonium carbonate and acetonitrile, and then the gel pieces were completely dried under reduced-pressure. Appropriate volumes of trypsin solution ( $25 \mu \mathrm{g} / \mu \mathrm{l}$ trypsin in $50 \mathrm{mM} \mathrm{NH}_{4} \mathrm{HCO}_{3}$ ) were added to the dried gel pieces. After an overnight incubation at $37^{\circ} \mathrm{C}$, the supernatant containing digested peptides was transferred to a new centrifuge tube. The gel pieces were washed with $20 \mu 1$ of $20 \mathrm{mM} \mathrm{NH}_{4} \mathrm{HCO}_{3}$ and $20 \mu \mathrm{l}$ of $50 \%$ (v/v) acetonitrile in $5 \%$ (v/v) formic acid. The peptide mixture was purified by using ZipTip pipette tips containing $\mathrm{C} 18$ reversed-phase material, according to the manufacturer's instructions. The eluted peptide solution was placed on a MALDI target plate, mixed with a matrix 2,5-dihydroxybenzoic acid (Sigma), and analyzed by a MALDI-Qq-TOF MS/MS QSTAR Pulsar $i$ (Applied Biosystems) in the linear positive ion mode. The spectrum data were submitted for protein identification with peptide mass fingerprinting, and database searching was performed with MASCOT ver1.9 (Matrix Science) in NCBInr.

Plasmid construction and transfectoin. The cDNAs that encode the N-terminus (amino acids: 1-278), middle section (amino acids: 264-531), and C-terminus (amino acids: 532-800) of mouse hnRNP U were amplified from the RNA of NIH 3T3 cells by RT-PCR. The primer set for the N-terminus was 5'-TTCTGCAGAGTTCTTCGCCTGTTAATGT-3' (forward) and 5'-TGTCTCACAGTTGCATGTTTTTATT-3' (reverse). The primer set for the middle section was 5'-TCTGCAG GTTTGTCTTGATACTTATAATTG-3' (forward) and 5'-AA GTCGACCAACAGTGTGTTCAGTTTTCC-3' (reverse). The primer set for the C-terminus was 5'-TTCTGCAGCAGA GAGCCCCACAGT-3' (forward) and 5'-AGTCGACATA ATATCCTTGGTGATAATGC-3' (reverse). The forward primers contain Pst I sites (underlined), and the reverse primers contain SalI sites (underlined). The RT-PCR products were cloned into the pTA2 vector (Toyobo, Osaka, Japan). To construct the cytomegalovirus expression vectors for deletion mutants after confirming the sequences, the $\mathrm{N}$ terminus fragment was excised from the pTA2 vector with $P s t \mathrm{I}$ and $X h o \mathrm{I}$ and inserted into the Pst $\mathrm{I} / X h o \mathrm{I}$ sites of the $\mathrm{pCMV} / \mathrm{myc} / \mathrm{nuc}$ vector (Invitrogen), which has a myc epitope tag and nuclear localization signal. Then, the XhoI site was blunted and re-ligated. The fragments for the middle section and the C-terminus of hnRNP U were excised with PstI and SalI and inserted into the same sites of the $\mathrm{pCMV} / \mathrm{myc} / \mathrm{nuc}$ vector. The expression vector for wild-type hnRNP U (amino acids: 1-800) was constructed by first inserting a carboxyl terminal-half fragment digested with PstI and SalI into the Pst $\mathrm{I} / \mathrm{XhoI}$ sites of the pCMV vector, followed by re-ligating the N-terminus fragment into the Pst I/XhoI-cut sites of the first construct. Ten micrograms each of the empty vectors and expression vectors was used to transfect Cos-7 cells with Lipofectamine 2000 (Invitrogen).

Immunoprecipitation and immunoblot. Subconfluent cultures of NIH 3T3 fibroblasts were serum-starved for 3 days and then growth stimulated by adding serum for $3 \mathrm{~h}$. Nuclear extracts $(30 \mu \mathrm{g})$ were incubated with the indicated antibodies $(2 \mu \mathrm{g}$ each) in $50 \mu \mathrm{l}$ of binding solution $(20 \mathrm{mM}$ Tris- $\mathrm{HCl} \mathrm{pH} 7.9$, $60 \mathrm{mM} \mathrm{KCl}, 1 \mathrm{mM}$ EDTA, 0.1\% Triton X-100, 1 mM DTT, $0.2 \mathrm{mg} / \mathrm{ml}$ bovine serum albumin, $10 \mu \mathrm{g} / \mathrm{ml}$ leupeptin) for $2 \mathrm{~h}$ at $4{ }^{\circ} \mathrm{C}$. Then, the antigen-antibody complexes were collected by incubation with $5 \mu \mathrm{l}$ of protein $\mathrm{G}$ magnetic bead suspension (New England Biolabs) for $1 \mathrm{~h}$ at room temperature and applied to a magnetic field for $5 \mathrm{~min}$ at $4^{\circ} \mathrm{C}$. Protein complexes were washed once with low-salt ChIP wash buffer $(20 \mathrm{mM}$ Tris- $\mathrm{HCl} \mathrm{pH} 8.1,150 \mathrm{mM} \mathrm{NaCl}, 2$ mM EDTA, $1 \%$ Triton $\mathrm{X}-100,0.1 \% \mathrm{SDS})$, twice with the high-salt wash buffer (500 $\mathrm{mM} \mathrm{NaCl})$, and once with TE buffer (10 mM Tris- $\mathrm{HCl}$, pH 8.0, 1 mM EDTA). Then, immunoprecipitates were resolved and probed with anti-hnRNP U (Bethyl Laboratories), anti-c-Myc (N-262; Santa Cruz) and anti-Max (C-124; Santa Cruz) rabbit polyclonal antibodies.

Nuclear extracts prepared from Cos-7 cells transfected with the expression vectors were immunoprecipitated with the anti-Myc epitope mouse monoclonal antibody. HnRNP U immunoprecipitations were resolved and probed with an antiMyc epitope mouse monoclonal antibody (PL14; Medical and Biological Laboratories, Nagoya, Japan), anti-c-Myc mouse monoclonal (9E10; Santa Cruz) and anti-Max rabbit polyclonal (C-124; Santa Cruz) antibodies.

RNA interference. A mixture of three short interfering RNA duplexes against mouse Hnrnpu (SASI_Mm01_00179155, SASI_Mm01_00179156,SASI_Mm01_00179157: Sigma Aldrich Japan) were applied by transfection at a final concentration of $10 \mathrm{nM}$ each, and $30 \mathrm{nM}$ of control short interfering RNA-A (Santa Cruz) was used as a control. Transfection of the short interfering RNAs was performed with TransIT-TKO transfection reagent (Mirus Bio), according to the manufacturer's protocol. After a 48-h transfection with hnRNP U and control short interfering RNAs, the cells were subjected to serum-starvation for 3 days and then restimulated for 6-36 h. Total cell lysates were subjected to immunoblot analysis with anti-hnRNP U (Bethyl Laboratories), anti-cMyc (N-262; Santa Cruz) and anti-Max (C-124; Santa Cruz) rabbit polyclonal antibodies, and anti- $\beta$-actin mouse monoclonal (AC-15; Sigma) antibody. Actin was used as a loading control. RT-PCR was performed by using the following set of primers: $O D C$ forward, 5'-CATCCAAAGGCAAAGT TGGT-3', reverse, 5'-AGCCACCACCAATATCAAGC-3'; ß-actin forward, 5'-CAGGGTGTGATGGTGGG-3', reverse, 5'-GGTCATCTTTTCACGGTTGG-3'. For cell proliferation 

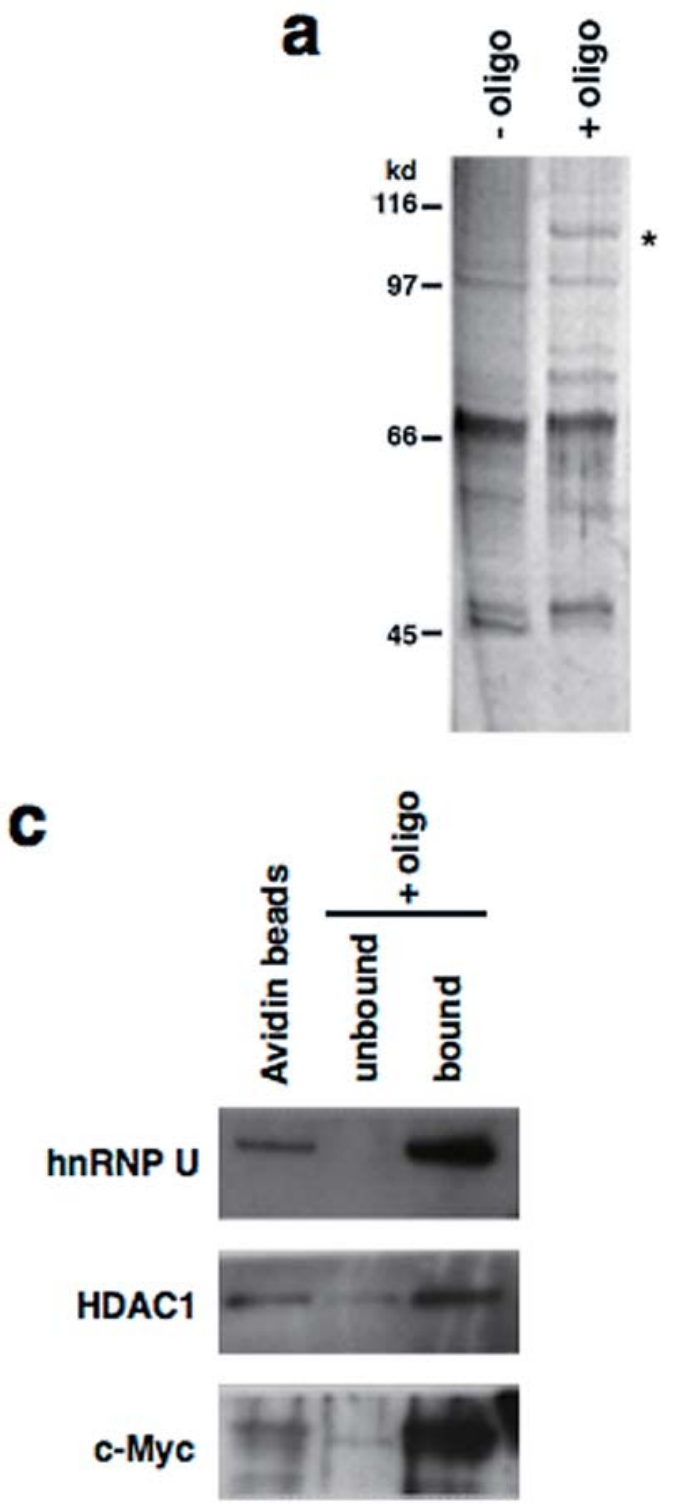

assays, 1500 cells/well were replated in 96 well plates and counted at 18 and $36 \mathrm{~h}$ after serum stimulation using a 2-(2methoxy-4-nitrophenyl)-3-(4-nitrophenyl)-5-(2,4-disulfophenyl)-2H-tetrazolium (WST-8) reduction assay kit (Wako Pure Chemicals, Osaka, Japan) according to the manufacturer's protocol. Essentially the same data were obtained at both time points.

Electrophoretic mobility-shift assay. Nuclear extracts from the serum-stimulated NIH 3T3 cells were utilized. The binding reaction was performed in a volume of $20 \mu \mathrm{l}$ consisting of $10 \mathrm{mM}$ Tris- $\mathrm{HCl} \mathrm{pH} 7.4,50 \mathrm{mM}$ potassium chloride, $2.5 \%$ glycerol, $1 \mathrm{mM}$ EDTA, $5 \mathrm{mM} \mathrm{Na} \mathrm{VO}_{4}, 1 \mu \mathrm{M}$ okadaic acid (Wako Pure Chemical Industries, Osaka, Japan), 0.05\% NP-40, and $50 \mathrm{ng}$ poly $(\mathrm{dI} \cdot \mathrm{dC})$ (Sigma-Aldrich Japan). Five hundred nanograms of 3T3 nuclear extract was added to the reaction mixture followed by 10-20 fmoles of a probe (sequence described below) that was biotin-labeled at the 3' ends of E-box1-inter. The DNA-binding reaction was allowed to proceed at room temperature for $30 \mathrm{~min}$. The complexes were analyzed by electrophoresis through a $5 \%$ acrylamide nondenaturing gel in $50 \mathrm{mM}$ Tris base, $50 \mathrm{mM}$ borate, and $1 \mathrm{mM}$ EDTA. The biotin-labeled probes were transferred to b

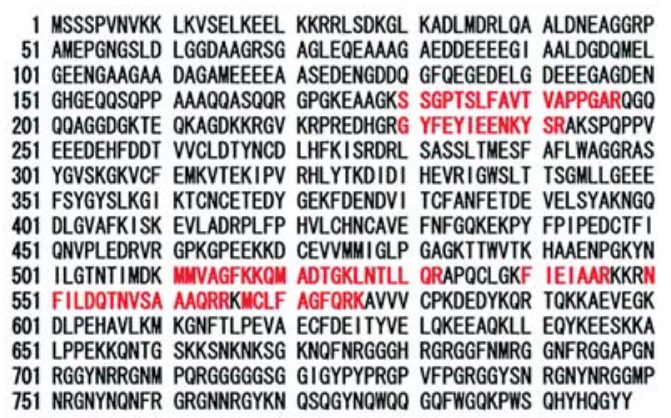

Figure 1. HnRNP U binds to E-box-containing region of $O D C$ promoter. (a) Silver staining of eluates from DNA-affinity chromatography containing the E-box flanking region of the rat $O D C$ promoter (+oligo) or from control streptavidin beads (-oligo). The band subjected to mass spectrometry is indicated by an asterisk. (b) Identification of a 110-kDa protein that binds to the E-box promoter by mass spectrometry. The obtained peptides are highlighted in red. (c) Immunoblot analysis of E-box-binding proteins Nuclear extracts of mouse NIH 3 T3 fibroblasts were prepared as described in Materials and methods. Proteins in the flow through (unbound) or $1 \mathrm{M}$ $\mathrm{NaCl}$ eluates (bound) from the resin were resolved and probed with antihnRNP U, anti-HDAC1 and anti-c-Myc rabbit polyclonal antibodies.

Amersham Hibond-N+ (GE Healthcare, UK) and detected with Chemiluminescent nucleic acid detection module (Pierce Biotechnology) according to manufacturer's instructions. When specified, a 200-fold molar excess of competitors was pre-incubated in the reaction mixture at room temperature for 30 min before adding the probe. The sequences of the competitors are: E-box 1, 5'-TGTGCGGCCACGTGTCGCG AGG-3'; E-box2, 5'-GCAGGGGACACGTGGCCGGAGG-3'; E-box1-inter, 5'-TGTGCGGCCACGTGTCGCGAGGCCC GGACTGCGGTCGCCGGCAGG-3'; Inter-E-box2, 5'-CGA GGCCCGGACTGCGGTCGCCGGCAGGGGACACGTGG CCGGAGG-3; and Inter, 5'-CGAGG CCCGGACTGCGGT CGCCGGCAGG-3'. For supershift experiments, $2 \mu \mathrm{g}$ of anti-hnRNP U (Bethyl Laboratories), anti-c-Myc (N-262; Santa Cruz) rabbit polyclonal antibodies or normal rabbit $\mathrm{IgG}$ (Upstate) were pre-incubated overnight at $4^{\circ} \mathrm{C}$ followed by the addition of the probe.

\section{Results}

hnRNP $U$ binds to E-box-containing promoter region of $O D C$ gene. Biotin-labeled sense and anti-sense oligonucleotides corresponding to the E-box region of the rat $O D C$ gene were 

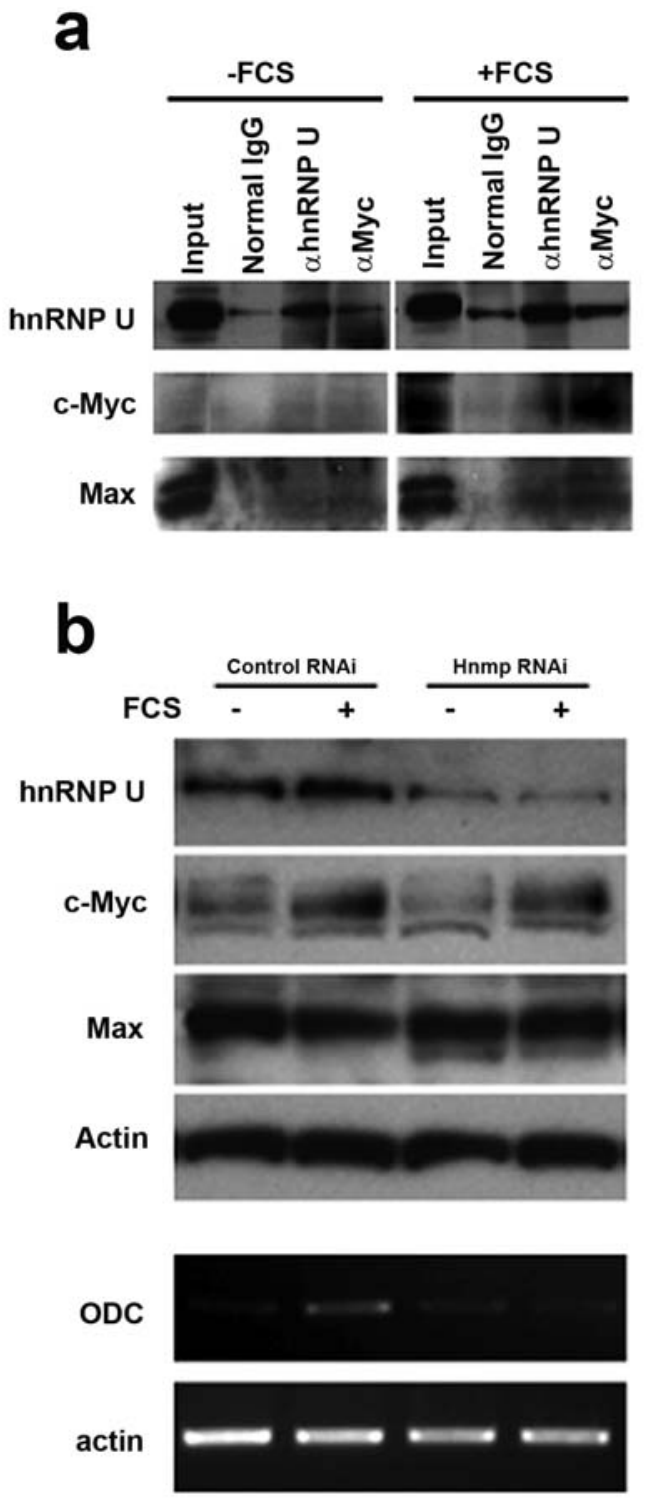

synthesized, annealed, and incubated with nuclear protein extracts prepared from rat (3Y1) or mouse (NIH 3T3) fibroblasts. E-box-bound proteins were recovered by incubation with avidin beads and eluted with $1 \mathrm{M} \mathrm{NaCl}$. One of the most intense bands migrating at $\sim 110 \mathrm{kDa}$ (Fig. 1a) was excised, in-gel-digested with trypsin, and subjected to MALDITOF/MS analysis. Six peptide sequences were obtained and matched with rat hnRNP U (Fig. 1b). Immunoblot analysis of $1 \mathrm{M} \mathrm{NaCl}$ eluates derived from mouse fibroblasts also showed binding of hnRNP $\mathrm{U}$ as well as HDAC1, c-Myc (Fig. 1c), and Max (data not shown) to the E-box DNA.

Association of hnRNP U with c-Myc-Max complex is essential for ODC gene induction by growth stimulation. Since binding of the c-Myc-Max complex to the E-box has a central role for $O D C$ expression induced by growth stimuli, we initially analysed the interaction between hnRNP $U$ and the c-MycMax complex under physiological condition. We used antihnRNP U antibody to immunoprecipitate proteins from nuclear extracts of 3T3 fibroblasts. Extracts from serum-stimulated cells contained greater amounts of c-Myc and Max compared with extracts from serum-starved cells (Fig. 2a). Similar

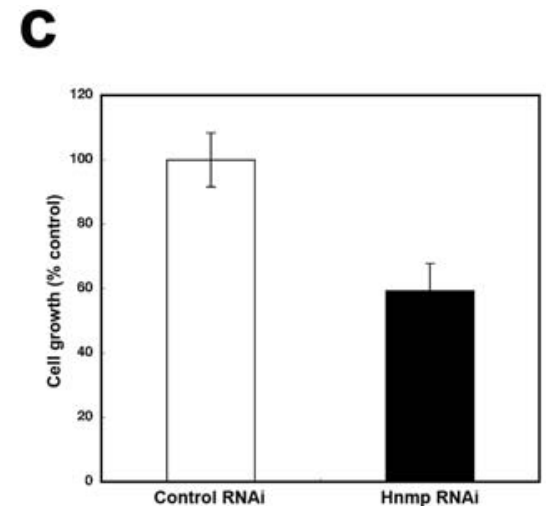

Figure 2. Physical and functional interactions between hnRNP U and c-Myc-Max heterodimers in mouse fibroblasts. (a) Reciprocal endogenous immunoprecipitation of hnRNP U and c-Myc-Max complexes before (-FCS) and after (+FCS) serum-stimulation. Subconfluent cultures of NIH 3T3 fibroblasts were serum-starved for 3 days and then growth stimulated by adding serum for $3 \mathrm{~h}$. (b) Effect on $O D C$ expression with down-regulation of hnRNP U. Upper and lower panels are immunoblot and RT-PCR data, respectively. Total cell lysates were subjected to immunoblot analysis with anti-hnRNP U, anti-c-Myc and anti-Max rabbit polyclonal antibodies, and anti- $\beta$-actin mouse monoclonal antibody. Actin was used as a loading control. (c) Effect on cell growth with down-regulation of hnRNP U. The cells treated with control siRNAs (open column) or Hnrnp siRNA (solid column) were growth stimulated for $36 \mathrm{~h}$ after serum starvation.

results were obtained by using anti-c-Myc antibody (Fig. 2a). Chromatin immunoprecipitation experiments confirmed that more hnRNP U and c-Myc-Max complexes were bound to the E-box of the $O D C$ gene after growth stimulation (data not shown).

To further test for function of hnRNP $U$ in the regulation of the $O D C$ gene through interaction with the c-Myc-Max complex, we used RNA interference to silence the hnRNP $U$ gene. 3T3 cells were transfected with a mixture of three short interfering RNAs against target sequences on the hnRNP $U$ gene. An almost complete and specific shut down of the gene resulting in very low endogenous hnRNP U expression was observed $36 \mathrm{~h}$ after transfection (Fig. 2b, upper panels). 3T3 cells were then serum-starved for an additional 3 days and growth-stimulated for $6 \mathrm{~h}$ by adding serum. No induction of $O D C$ was observed in the hnRNP $U$-silenced cells after addition of serum, whereas the expression of the gene was up-regulated in cells transfected with control short interfering RNAs (Fig. 2b, lower panels). In addition, down-regulation of $h n R N P U$ resulted in suppression of cell growth after the stimulation (Fig. 2c). The protein levels of c-Myc and Max in the hnRNP $U$-silenced cells were unaffected (Fig. 2b, upper 
a

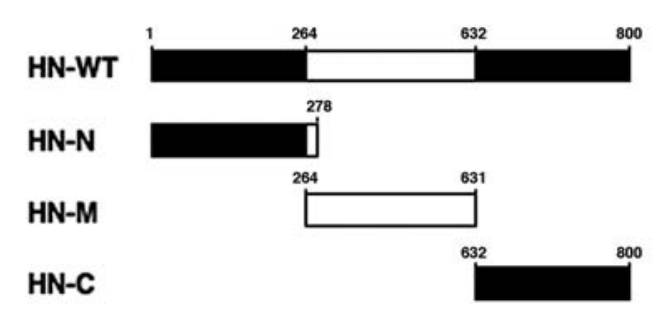

b

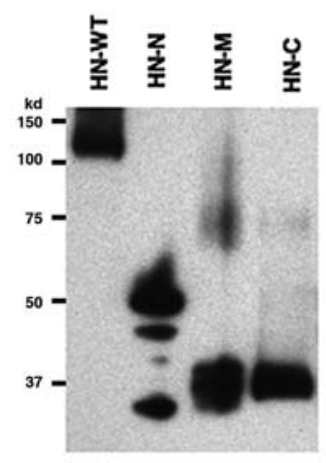

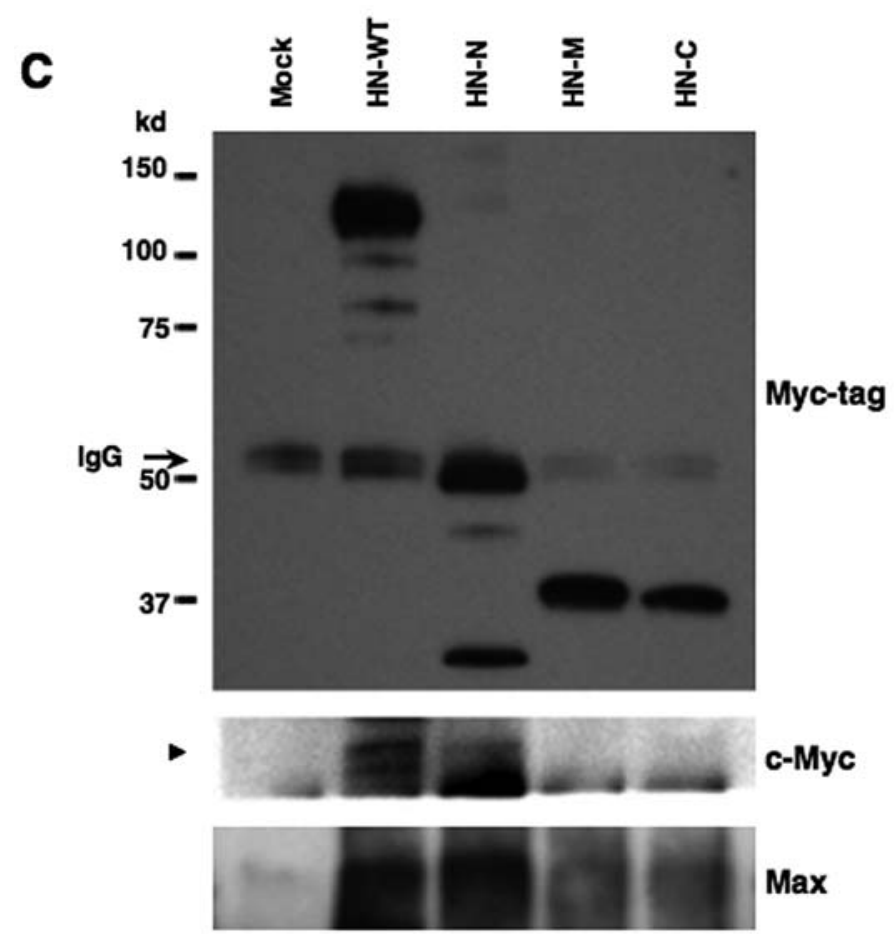

panels). These results suggest that hnRNP $\mathrm{U}$ is indispensable for the induction of $O D C$ and cell proliferation by the c-MycMax complex through binding to the E-box.

The N-terminal domain of hnRNP $U$ is sufficient to mediate its association with c-Myc-Max complex. The multifunctional properties of hnRNP U have been mapped to specific domains within the protein (Fig. 3a). To determine which domain of hnRNP U interacts with the c-Myc-Max complex, Myc epitope-tagged hnRNP U proteins were expressed in Cos-7 cells (Fig. 3b). Nuclear extracts from Cos-7 cells transfected with the Myc epitope-tagged hnRNP U constructs were immunoprecipitated with the anti-Myc epitope antibody and probed by immunoblot with the anti-c-Myc or the anti-Max antibodies. Full-length and N-terminal domain hnRNP U proteins were co-immunoprecipitated with c-Myc and Max, demonstrating that the N-terminal domain is sufficient to mediate the interaction of hnRNP $U$ with the c-Myc-Max complex (Fig. 3c). We are currently investigating which domains of c-Myc and/or Max interact with the N-terminal domain and whether c-Myc-Max heterodimerization is needed for the interaction.
Figure 3. Identification of a domain responsible for association of hnRNP U with the c-Myc-Max complex. (a) Schematic representation of Myc epitopetagged hnRNP U expression constructs. (b) Immunoblot data of whole cell lysates derived from Cos-7 cells transfected with the Myc epitope-tagged hnRNP U constructs. The lysates were resolved and probed with the antiMyc epitope mouse monoclonal antibody. (c) Nuclear extracts prepared from transfected Cos-7 cells were immunoprecipitated with the anti-Myc epitope mouse monoclonal antibody. HnRNP U immunoprecipitations were resolved and probed with the anti-Myc epitope mouse monoclonal (Myctag), anti-c-Myc mouse monoclonal and anti-Max rabbit polyclonal antibodies. The arrowhead indicates c-Myc protein bands. HN-WT, full length hnRNP U; HN-N, N-terminal of hnRNP U; HN-M, middle section of hnRNP U; HN-C, C-terminal of hnRNP U.

Interaction between hnRNP $U$ and c-Myc-Max complex on E-box-containing promoter region. We performed electrophoretic mobility-shift assays to narrow down the binding region for hnRNP $\mathrm{U}$ in the $O D C$ promoter. Two shifted bands were observed when DNA fragments containing the first E-box and 31 bps of the area between the E-boxes were used as a probe (Fig. 4a and b). Supershift assays with anti-hnRNP U and anti-c-Myc antibodies revealed that the upper and lower bands corresponded to c-Myc-Max and hnRNP U-containing complexes, respectively (Fig. 4b). The lower band was specifically abolished in the presence of competitor fragments containing the area between the two E-boxes.

DNA fragments containing the inner part and either one of the E-boxes interfered with the formation of both c-MycMax and hnRNP U-containing complexes. Interestingly, each of the E-box fragments somewhat diminished the formation of hnRNP U-containing complexes (the first E-box fragment had a stronger effect), in addition to completely inhibiting the binding of c-Myc-Max complexes to the probe (Fig. 4b). Moreover, decreased intensities for the upper and lower bands in the supershift assays using anti-hnRNP U and antic-Myc antibodies, respectively, were obvious although single 
a

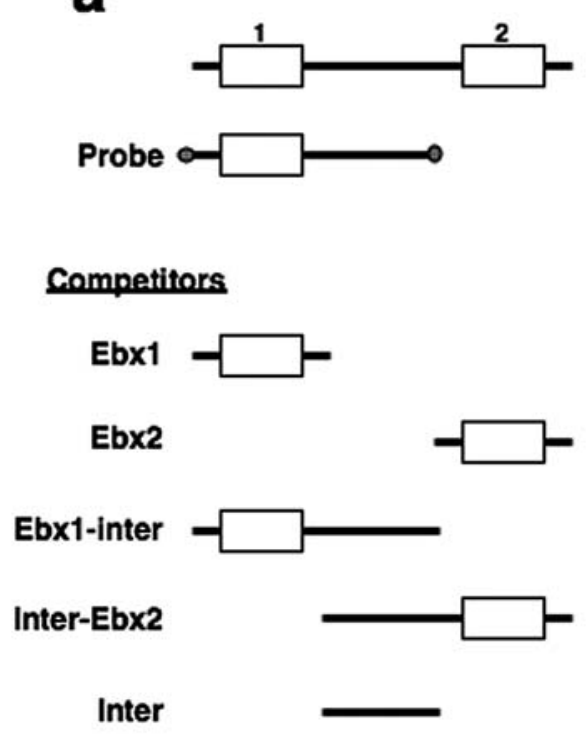

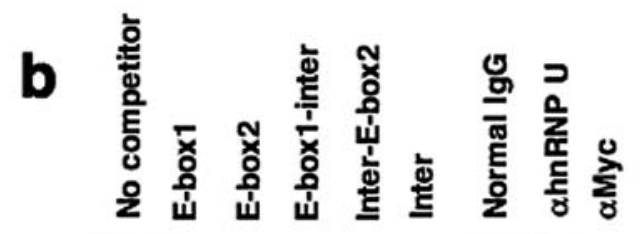

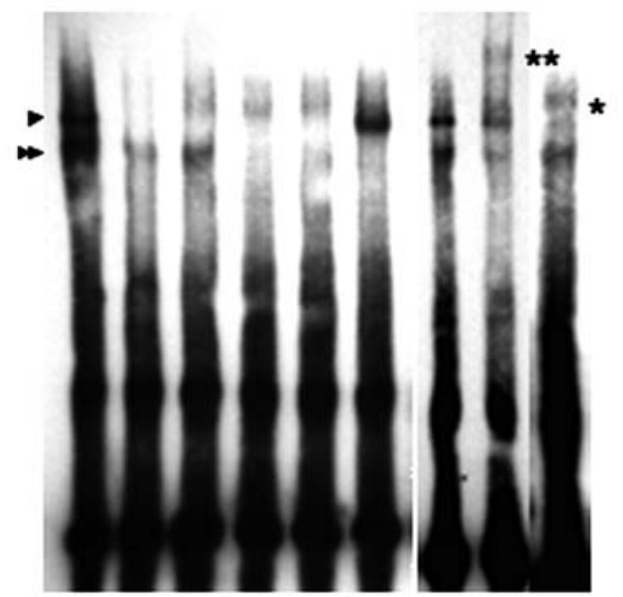

C

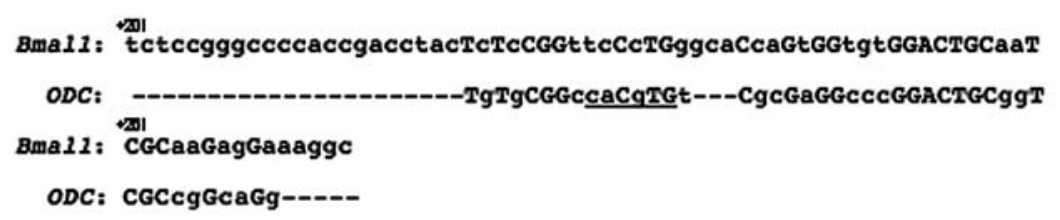

Figure 4. Identification of a putative binding site of hnRNP U on E-box-containing $O D C$ promoter region. (a) Schematic representation of probe and competitor fragments used in EMSA assays. The rat $O D C$ promoter contains two E-boxes (Ebx1 and Ebx2) separated by 34 bps (Inter). (b) Interaction between hnRNP U and the c-Myc-Max complex on the E-box-containing promoter region. The arrowhead and double-arrowhead correspond to c-Myc-Maxand hnRNP U-containing complexes, respectively. The c-Myc-Max-containing complexes and the hnRNP U-containing complexes are supershifted by the anti-c-Myc (asterisk) and anti-hnRNP U (double-asterisk) rabbit polyclonal antibodies, respectively. (c) Putative hnRNP U-binding sequence. Alignment of the sequences identified as the hnRNP U-binding regions of Bmall and ODC promoters revealed a 13-nucleotide segment as a putative hnRNP U-binding sequence. Capital letters indicate the identical nucleotides between the two regions, and E-box1 is underlined. The transcription initiation site of the longest product of Bmall is designated as nucleotide +1 .

major shifted-bands were only observed in each sample (Fig. 4b). Thus, hnRNP U may mainly contact the segment between the E-boxes, with an additional weaker interaction to the first E-box probably through association with the c-MycMax complex.

The rigid structure at the E-box-flanking region might not be the nuclear matrix because the region is composed of GC-rich sequences, whereas the DNA regions that anchor chromosomal DNA to the nuclear matrix are usually AT rich (16). Binding of hnRNP $U$ to GC-rich sequences is not exceptional for the $O D C$ promoter since the GC-rich region (nucleotides -27 to +266 ) in the Bmall promoter was recently identified as the binding site of hnRNP U, binding of which is responsible for circadian expression of the gene (17). Comparison of these promoter regions revealed a 13-nucleotide segment of the putative hnRNP U-binding sequence, GGACTGCRRTCGC (Fig. 4c). This stretch corresponds to +251 to +263 of the Bmall promoter that is included in one of the four important sites identified for hnRNP $U$ binding (17).

\section{Discussion}

In the present study, we show that hnRNP U physically (Figs. 2 and 3) and functionally (Fig. 2) interacts with the c-Myc-Max complex. hnRNP U interacts with transcription factors including RNA polymerase II (2), TFII H (8), and WT1 (3) as well as histone acetyltransferases $(18,19)$. On the other hand, c-Myc controls RNA polymerase II- and IIIregulated gene transcription $(20,21)$. Furthermore, reciprocal interactions between c-Myc-Max complex and histone acetyltransferases $(22,23)$ and between Mxds/Mnt-Max complex and histone deacetylases $(13,14)$ have been established. Therefore, it is not surprising that hnRNP U and the c-MycMax complex interact on the E-box-containing region of the $O D C$ promoter.

This study provides the first direct evidence to connect the Myc-regulated proliferation pathway and a molecule, hnRNP U, involved in the circadian molecular oscillator. In this regard, it is intriguing that the Clock-Bmall heterodimer, the positive loop of the circadian clock, binds to E-box and 
E-box-like enhancer sequences to mediate the transcription of certain genes in cooperation with Myc family proteins $(24,25)$. Nevertheless, the association of hnRNP U with the c-Myc-Max complex is essential for $O D C$ gene induction by growth stimulation (Fig. 2). HnRNP U might be a component of another cascade that integrates the circadian clock with cell cycle regulation and tumorigenesis, which is a rapidly growing area of research.

\section{Acknowledgements}

This work was supported in part by a Grant-in-Aid for Scientific Research on Priority Areas (KAKENHI) from the Ministry of Education, Culture, Sports, Science and Technology of Japan.

\section{References}

1. Howell M, Borchers C and Milgram SL: Heterogeneous nuclear ribonuclear protein $\mathrm{U}$ associates with YAP and regulates its coactivation of Bax transcription. J Biol Chem 279: 26300-26306, 2004.

2. Kukalev A, Nord Y, Palmberg C, Bergman T and Percipalle P: Actin and hnRNP U cooperate for productive transcription by RNA polymerase II. Nat Struct Mol Biol 12: 238-244, 2005.

3. Spraggon L, Dudnakova T, Slight J, et al: hnRNP-U directly interacts with WT1 and modulates WT1 transcriptional activation. Oncogene 26: 1484-1491, 2007.

4. Jackson DA, Dickinson P and Cook PR: The size of chromatin loops in HeLa cells. EMBO J 9: 567-571, 1990.

5. von Kries JP, Buck F and Stratling WH: Chicken MAR binding protein p120 is identical to human heterogeneous nuclear ribonucleoprotein (hnRNP) U. Nucleic Acids Res 22: 1215-1220, 1994.

6. Pinol-Roma S, Choi YD, Matunis MJ and Dreyfuss G: Immunopurification of heterogeneous nuclear ribonucleoprotein particles reveals an assortment of RNA-binding proteins. Genes Dev 2: 215-227, 1988

7. Kiledjian M and Dreyfuss G: Primary structure and binding activity of the hnRNP U protein: binding RNA through RGG box. EMBO J 11: 2655-2664, 1992.

8. Kim MK and Nikodem VM: hnRNP U inhibits carboxyterminal domain phosphorylation by TFIIH and represses RNA polymerase II elongation. Mol Cell Biol 19: 6833-6844, 1999.

9. Eisenman RN: Deconstructing myc. Genes Dev 15: 2023-2030, 2001.

10. Hurlin PJ, Queva C and Eisenman RN: Mnt: a novel Maxinteracting protein and Myc antagonist. Curr Top Microbiol Immunol 224: 115-121, 1997.
11. Grandori C, Cowley SM, James LP and Eisenman RN: The Myc/Max/Mad network and the transcriptional control of cell behavior. Annu Rev Cell Dev Biol 16: 653-699, 2000.

12. Zhou ZQ and Hurlin PJ: The interplay between Mad and Myc in proliferation and differentiation. Trends Cell Biol 11: S10-S14, 2001.

13. Ayer DE: Histone deacetylases: transcriptional repression with SINers and NuRDs. Trends Cell Biol 9: 193-198, 1999.

14. Hurlin PJ and Huang J: The MAX-interacting transcription factor network. Semin Cancer Biol 16: 265-274, 2006.

15. Matsuoka Y, Fukamachi K, Uehara N, Tsuda $\mathrm{H}$ and Tsubura A: Induction of a novel histone deacetylase 1/c-Myc/Mnt/Max complex formation is implicated in parity-induced refractoriness to mammary carcinogenesis. Cancer Sci 99: 309-315, 2008.

16. Cockerill PN and Garrard WT: Chromosomal loop anchorage of the kappa immunoglobulin gene occurs next to the enhancer in a region containing topoisomerase II sites. Cell 44: 273-282, 1986.

17. Onishi Y, Hanai S, Ohno T, Hara Y and Ishida N: Rhythmic SAF-A binding underlies circadian transcription of the Bmall gene. Mol Cell Biol 28: 3477-3488, 2008.

18. Martens JH, Verlaan M, Kalkhoven E, Dorsman JC and Zantema A: Scaffold/matrix attachment region elements interact with a p300-scaffold attachment factor A complex and are bound by acetylated nucleosomes. Mol Cell Biol 22: 2598-2606, 2002.

19. Obrdlik A, Kukalev A, Louvet E, Farrants AK, Caputo L and Percipalle P: The histone acetyltransferase PCAF associates with actin and hnRNP U for RNA polymerase II transcription. Mol Cell Biol 28: 6342-6357, 2008.

20. Oskarsson T and Trumpp A: The Myc trilogy: lord of RNA polymerases. Nat Cell Biol 7: 215-217, 2005.

21. Adhikary S and Eilers M: Transcriptional regulation and transformation by Myc proteins. Nat Rev Mol Cell Biol 6: 635-645, 2005.

22. McMahon SB, Van Buskirk HA, Dugan KA, Copeland TD and Cole MD: The novel ATM-related protein TRRAP is an essential cofactor for the c-Myc and E2F oncoproteins. Cell 94: 363-374, 1998.

23. Vervoorts J, Luscher-Firzlaff JM, Rottmann S, et al: Stimulation of c-MYC transcriptional activity and acetylation by recruitment of the cofactor CBP. EMBO Rep 4: 484-490, 2003.

24. Kondratov RV, Shamanna RK, Kondratova AA, Gorbacheva VY and Antoch MP: Dual role of the CLOCK/BMAL1 circadian complex in transcriptional regulation. FASEB J 20: 530-532, 2006.

25. Sherman $\mathrm{H}$ and Froy O: Expression of human beta-defensin 1 is regulated via c-Myc and the biological clock. Mol Immunol 45: 3163-3167, 2008. 